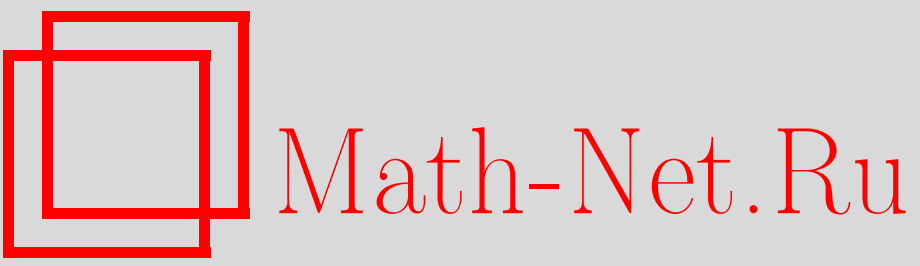

А. В. Горшков, Стабилизация одномерного уравнения теплопроводности на полуограниченном стержне, УМН, 2001, том 56, выпуск 2, 213-214

DOI: https://doi.org/10.4213/rm388

Использование Общероссийского математического портала Math-Net.Ru подразумевает, что вы прочитали и согласны с пользовательским соглашением

http://www.mathnet.ru/rus/agreement

Параметры загрузки:

IP : 54.197 .217 .227

26 апреля 2023 г., 12:41:14 


\title{
СТАБИЛИЗАЦИЯ ОДНОМЕРНОГО УРАВНЕНИЯ ТЕПЛОПРОВОДНОСТИ НА ПОЛУОГРАНИЧЕННОМ СТЕРЖНЕ
}

\author{
А. В. ГОРШКОВ
}

В последнее время активно исследовалась задача точной управляемости для широкого класса параболических уравнений и систем Навье-Стокса (см. [1], [2] и приводимьй там список литературы). Однако простой анализ показывает невозможность получения такого рода результатов в случае неограниченных областей даже для уравнения теплопроводности. Поэтому в случае неограниченных областей естественным является переход от задачи управляемости на конечном промежутке времени к задаче стабилизации при времени $t$, стремящемся к бесконечности. Эта задача состоит в следующем.

Рассматривается уравнение теплопроводности без внешних источников тепла на полуограниченном стержне:

$$
\begin{gathered}
\frac{\partial y(t, x)}{\partial t}-\frac{\partial^{2} y(t, x)}{\partial x^{2}}=0, \quad(t, x) \in \mathbb{R}_{+} \times \mathbb{R}_{+} \equiv \Omega, \\
\left.y\right|_{t=0}=y_{0}(x), \quad y_{0}(x) \in Y_{0}^{\alpha}, \quad \alpha \in(0,1),
\end{gathered}
$$

где $Y_{0}^{\alpha}=\left\{y_{0}(x) \mid y_{0} \in C^{2}(0, \infty), e^{\alpha x} y_{0}(x) \in L_{1}(0, \infty)\right\}$.

Требуется для каждого натурального $k$ найти такое граничное условие (управление) $u_{k}(t)$, чтобы для решения $y(t, x)$ задачи $(1),(2)$ с граничным условием

$$
\left.y\right|_{x=0}=u_{k}(t)
$$

при любом фиксированном $x \in(0, \infty)$ выполнялось следующее условие стабилизации:

$$
t^{k} y(t, x) \in L_{2}(0, \infty)
$$

Введем обозначения: $H^{1,2}\left(\mathbb{R}_{+}^{2}\right)$ - пространство Соболева функций $y(t, x)$ с нормой

$$
\|y\|_{H^{1,2}\left(\mathbb{R}_{+}^{2}\right)}^{2}=\|y\|_{L^{2}\left(\mathbb{R}_{+}^{2}\right)}^{2}+\left\|\partial_{t} y\right\|_{L^{2}\left(\mathbb{R}_{+}^{2}\right)}^{2}+\left\|\partial_{x x} y\right\|_{L^{2}\left(\mathbb{R}_{+}^{2}\right)}^{2},
$$

$H^{\frac{3}{4}}\left(\mathbb{R}_{+}\right)$- пространство Соболева функций $u(t)$, квадратично суммируемых с дробной производной порядка $\frac{3}{4}$ (см. [3]).

Tеорема. Для любых $y_{0}(x) \in Y_{0}^{\alpha}, k \in \mathbb{N}$ существует решение $\left(y_{k}(t, x), u_{k}(t)\right) \in$ $H^{1,2}(\Omega) \times H^{\frac{3}{4}}(0, \infty)$ задачи $(1)-(4)$.

СХема доКАЗАТЕЛЬСТВА. Будем решать недоопределенную задачу $(1),(2)$, и среди всех ее решений выделим те, которые удовлетворяют (4). Ограничение найденных функций при $x=0$ даст искомое граничное управление.

С помощью преобразования Лапласа $\widehat{y}(\tau, x)=\int_{0}^{\infty} e^{-\tau t} y(t, x) d t$ сведем нашу задачу к обькновенному дифференциальному уравнению с параметром $\tau$ :

$$
\partial_{x x}^{2} \widehat{y}(\tau, x)-\tau \widehat{y}(\tau, x)=-y_{0}(x) .
$$

Если $y(t, x) \in H^{1,2}(\Omega)$, то $\widehat{y}(\tau, x)$ является аналитической функцией в комплексной области $D=\{\tau \in \mathbb{C} \mid \operatorname{Re} \tau>0\}$. Общее решение уравнения (5) в классе ограниченных функций по $x$ и $\tau$ имеет вид:

$$
\begin{aligned}
\widehat{y}(\tau, x)= & \widehat{u}(\tau) e^{-\sqrt{\tau} x}-\frac{e^{-\sqrt{\tau} x}}{2 \sqrt{\tau}} \int_{0}^{\infty} e^{-\sqrt{\tau} z} y_{0}(z) d z \\
& +\frac{e^{-\sqrt{\tau} x}}{2 \sqrt{\tau}} \int_{0}^{x} e^{\sqrt{\tau} z} y_{0}(z) d z+\frac{e^{\sqrt{\tau} x}}{2 \sqrt{\tau}} \int_{x}^{\infty} e^{-\sqrt{\tau} z} y_{0}(z) d z .
\end{aligned}
$$


Здесь $\widehat{u}(\tau)$ - аналитическое в области $\left\{\tau \mid \operatorname{Re} \tau>-\varepsilon_{0}, \varepsilon_{0}>0\right\}$ преобразование Лапласа произвольной функции из $H^{\frac{3}{4}}(0, \infty)$. Тогда из $(6)$ следует, что общее решение $\widehat{y}(\tau, x)$ допускает аналитическое продолжение в область $\mathbb{C} \backslash\left(\mathbb{R}_{-} \cup\{0\} \cup\left\{\tau \mid \operatorname{Re} \tau \leqslant-\varepsilon_{0}\right\}\right)$.

Применяя к $\widehat{y}(t, x)$ обратное преобразование Лапласа, получим:

$$
y(t, x)=\frac{1}{2 \pi i} \int_{\gamma_{k_{0}}} e^{\tau t} \widehat{y}(\tau, x) d \tau,
$$

где интегрирование ведется по произволшной прямой $\gamma_{k_{0}}=\left\{\tau \mid \operatorname{Re} \tau=k_{0}>0\right\}$ в комплексной плоскости.

Интегрирование в (7) по прямой $\gamma_{k_{0}}$, согласно интегральной формуле Коши, сводится к интегрированию по кривой $\gamma_{-k_{1}, \varepsilon}$, состоящей из пяти элементов: $\gamma_{(-\infty,-\varepsilon)}=\left\{\tau \mid \operatorname{Re} \tau=-k_{1}\right.$, $\operatorname{Im} \tau<-\varepsilon\}, \gamma_{-\varepsilon}=\left\{\tau \mid \operatorname{Re} \tau \in\left(-k_{1}, 0\right), \operatorname{Im} \tau=-\varepsilon\right\}, \gamma_{(-\varepsilon, \varepsilon)}=\{\tau \mid \operatorname{Re} \tau=0, \operatorname{Im} \tau \in(-\varepsilon, \varepsilon)\}$, $\gamma_{\varepsilon}=\left\{\tau \mid \operatorname{Re} \tau \in\left(-k_{1}, 0\right), \operatorname{Im} \tau=\varepsilon\right\}, \gamma_{(\varepsilon, \infty)}=\left\{\tau \mid \operatorname{Re} \tau=-k_{1}, \operatorname{Im} \tau>\varepsilon\right\}$, где $k_{1}<\varepsilon_{0}$.

Здесь $\varepsilon>0$ - произвольный параметр интегрирования, контур обходится в направлении "снизу-вверх". Устремляя $\varepsilon$ к нулю и производя элементарные вычисления, получаем следующее выражение для решения уравнения теплопроводности:

$$
\begin{aligned}
y(t, x)= & -\frac{e^{-k_{1} t}}{2 \pi} \int_{-\infty}^{\infty} e^{i \xi t} \widehat{y}\left(-k_{1}+i \xi, x\right) d \xi \\
& +\frac{2}{\pi} \int_{0}^{\sqrt{k_{1}}} \sin (\lambda x)\left[\lambda \widehat{u}\left(-\lambda^{2}\right)+\int_{0}^{\infty} \sin (\lambda z) y_{0}(z) d z\right] e^{-\lambda^{2} t} d \lambda .
\end{aligned}
$$

Первое слагаемое в (8) убьвает экспоненциально. Положив $\widehat{u}(\tau)=\frac{P_{k-1}(\tau)}{\left(\tau+\varepsilon_{0}\right)^{4 k+4}}$, где $P_{k-1}(\tau)$ - некоторьй полином степени $k-1$, получим оценку $\lambda \widehat{u}\left(-\lambda^{2}\right)+\int_{0}^{\infty} \sin (\lambda z) y_{0}(z) d z \leqslant C \lambda^{2 k+1}$ при достаточно малых $\lambda$. Коэффициенты полинома $P_{k-1}(\tau)$ определяются из условия совпадения первых $k$ членов разложения функций $\lambda \widehat{u}\left(-\lambda^{2}\right)$ и $-\int_{0}^{\infty} \sin (\lambda z) y_{0}(z) d z$ по $\lambda$. Тогда второе слагаемое в (8) при достаточно больших $t$ оценивается через $\frac{C}{t^{k+1}}$ и представляет главную асимптотику решения $y(t, x)$ при $t \rightarrow \infty$.

\section{СПИСОК ЛИТЕРАТУРЫ}

[1] A. V. Fursikov, O. Yu. Imanuilov // Acta Appl. Math. 1994. V. 37. № 2. P. 67-76. [2] А. В. Фурсиков, О.Ю. Эмануилов // Матем. сб. 1996. Т. 187. №9. С. 103-138. [3] Ж.--Л. Лионс, Э. Мадженес. Неоднородные задачи и их приложения. М.: Мир, 1971. 